\title{
Impact of the confinement on the intra-cage dynamics of molecular hydrogen in clathrate hydrates
}

\author{
Margarita Russina, ${ }^{*}$, Ewout Kemner ${ }^{1, b}$ and Ferenc Mezei ${ }^{2,3, c}$ \\ ${ }^{1}$ Helmholtz Zentrum Berlin für Materialien und Energie, Hahn-Meitner-Platz 1,1049 Berlin, \\ Germany \\ ${ }^{2}$ HAS Wigner Research Center for Physics, Konkoly Thege ut 29-33, 1121 Budapest, Hungary \\ ${ }^{3}$ European Spallation Source ERIC, PO BOX 76, 22100 Lund, Sweden \\ amargarita.russina@helmholtz-berlin.de, bevout.kemner@helmholtz-berlin.de, \\ cferenc.mezei@esss.se
}

Keywords: clathrates, confinement, hydrogen storage, diffusive mobility, neutron spectroscopy

\begin{abstract}
We have studied the diffusive mobility of hydrogen molecules confined in different size cages in clathrate hydrates. In clathrate hydrate $\mathrm{H}_{2}$ molecules are effectively stored by confinement in two different size cages of the nano-porous host structure with accessible volumes of about 0.50 and $0.67 \mathrm{~nm}$ diameters, respectively. For the processes of sorption and desorption of the stored hydrogen the diffusive mobility of the molecules plays a fundamental role. In the present study we have focused on the dynamics of the $\mathrm{H}_{2}$ molecules inside the cages as one aspect of global guest molecule mobility across the crystalline host structure. We have found that for the two cage sizes different in diameter by only $34 \%$ and in volume by about a factor of 2.4 , the dimension can modify the diffusive mobility of confined hydrogen in both directions, i.e. reducing and surprisingly enhancing mobility compared to the bulk at the same temperature. In the smaller cages of clathrate hydrates hydrogen molecules are localized in the center of the cages even at temperatures $>100 \mathrm{~K}$. Confinement in the large cages leads to the onset already at $\mathrm{T}=10 \mathrm{~K}$ of jump diffusion between sorption sites separated from each other by about $2.9 \AA$ at the 4 corners of a tetrahedron. At this temperature bulk hydrogen is frozen at ambient pressure and shows no molecular mobility on the same time scale. A particular feature of this diffusive mobility is the pronounced dynamic heterogeneity: only a temperature dependent fraction of the $\mathrm{H}_{2}$ molecules was found mobile on the time scale covered by the neutron spectrometer used. The differences in microscopic dynamics inside the cages of two different sizes can help to explain the differences in the parameters of macroscopic mobility: trapping of hydrogen molecules in smaller pores matching the molecule size can to play a role in the higher desorption temperature for the small cages.
\end{abstract}

\section{Introduction}

Clathrates are nanostructured porous materials consisting of cages formed by ice, the host material, with potentially varying number of guest molecules confined in the cages (pores). Clathrates with different guests represent a growing class of new materials of actual interest both for fundamental research and application opportunities. They are typically synthetized at high gas pressures from ice and guest molecules of the pressurized, hydrophobic gas that weakly bind to water and rather promote the ice to form cages around them. It is important that the presence of host molecules stabilizes the structure of clathrates, which usually collapses in the process of guest deintercalation. In general this mechanism applies to formation of clathrates with many other gasses too, with the clathrate structure depending in particular on the size of the encapsulated guest.

Clathrate hydrates are a group of materials with potential for hydrogen storage, discovered first in 1999 [1]. The hydrogen filled clathrates form so-called structure sII, consisting of two types of cages, i.e. "small” dodecahedron $5^{12}$ cages with a mean crystallographic radius of $3.95 \AA$ (of which $2.5 \AA$ radius is accessible for the hydrogen gas) and "large" hexakaidecahedron $6^{4} 5^{12}$ cages with an average radius of $4.75 \AA$ (of which 3.35 accessible) [2], cf. Fig 1. While the small cage, as a rule, 
accommodates one $\mathrm{H}_{2}$ molecule, the large cage can accommodate 2-4 $\mathrm{H}_{2}$ molecules depending on the conditions applied. The cubic unit cell with $\mathrm{a}=17.047 \AA$ is formed by 8 large and 16 small cages. Thus, in the case of maximum occupancy, the clathrates can be denoted as $48 \mathrm{H}_{2} \mathrm{x} 136 \mathrm{H}_{2} \mathrm{O}$ [3]. Thus the maximal storage capacity of clathrate hydrates reaches $3.77 \mathrm{wt} \%$.

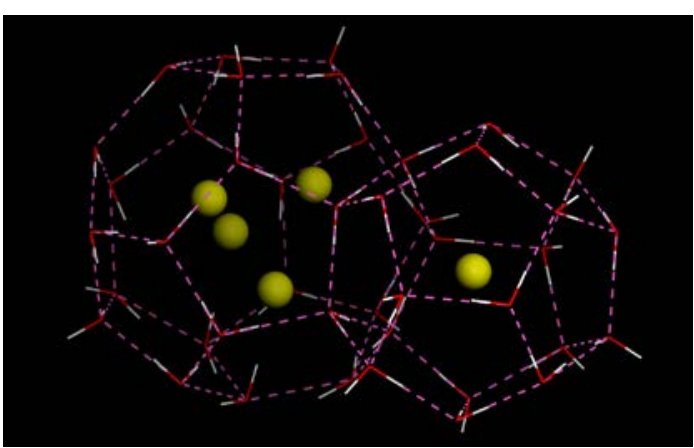

Fig. 1 Structure of hydrogen clathrates. Hydrogen molecule sites are indicated by the spheres, framework water molecules are at the vertices of the polyhedral shown by the lines. The clathrate of type sII is formed by 8 large cages of hexakaidecahedron $\left(6^{4} 5^{12}\right)$ and 16 small cages of dodecahedron $\left(5^{12}\right)$ shapes with a mean crystallographic radii of $4.73 \AA$ and $3.95 \AA$ A, respectively.

In pure clathrates, where $\mathrm{H}_{2}$ occupies both cages, the gas is loaded at 2000 bars at $250 \mathrm{~K}$ [3], [4]. Under ambient pressure the temperature stability of these clathrates is reduced to about $70 \mathrm{~K}$, i.e. the part of the gas load starts to leave the clathrate. Later it was discovered that the gas sorption pressure can be reduced and temperature stability under ambient pressure can be improved by introducing large promoter molecules into the large clathrate cages and hydrogen being only in the small ones. For instance, binary clathrates with tetrahydrofuran in the large cages [5] can be formed already at a pressure of 60 bar of $\mathrm{H}_{2}$ at $260 \mathrm{~K}$ and remain stable under ambient pressure to temperatures of up to $230 \mathrm{~K}$. The deintercalating gas molecules escape the clathrate by the way of diffusion from the inside of the cages to the surface (if they were bound/localized inside the cage) and through the pentagonal and/or hexagonal surfaces of the cages. The first of these two diffusive steps is characterized by the motion of the molecules inside the cages, which is the subject of the current study.

Neutron scattering is a powerful probe to study microscopic dynamics, in particular of hydrogen. The feature directly determined in a neutron scattering experiment is the double differential scattering cross section, which is proportional to the dynamic structure factor $S(\boldsymbol{Q}, \omega)$ with $\hbar \omega$ standing for the neutron energy transfer and $\boldsymbol{Q}=\boldsymbol{k}_{f}-\boldsymbol{k}_{i}$ for the neutron momentum transfer [6]. In the experiment we could selectively probe the motion of individual $\mathrm{H}_{2}$ molecules due to the very large incoherent scattering cross section of hydrogen compared to deuterated $\left(\mathrm{D}_{2} \mathrm{O}\right)$ ice.

High frequency dynamics of confined $\mathrm{H}_{2}$ was studied previously in tetrahydrofuran clathrates [7]. The existence of broad rotational and translational "rattling" modes, predicted first in 5D quantum molecular dynamic calculations [8], was found for hydrogen in the small cages. The experimental comparison of high frequency dynamics in large and small cages $[9,10]$ reveals striking differences of the "rattling" modes, a hint to the possible impact of the confinement. Migration of $\mathrm{H}_{2}$ between cages was studied in several works. Upper limits of activation barriers for $\mathrm{H}_{2}$ to pass through pentagonal and hexagonal rings on the cage surface was first determined theoretically [11] and was found to be 26 and $6 \mathrm{kcal} / \mathrm{mol}$, respectively. The various experimental studies did not lead yet to consistent values. [12], [13], [14], [15].

\section{Experimental}

The clathrate samples were prepared using fine deuterated ice powder by procedures described before in [16] and [17]. $99.8 \%$ deuterated ice was used in order to reduce the incoherent neutron scattering background from the clathrate matrix by more than an order of magnitude. The tetrahydrofuran promoter guest was also deuterated. Thus in all $\mathrm{H}_{2}$ loaded samples the incoherent scattering on the guest $\mathrm{H}_{2}$ molecules dominated the observed signal, with the exception of well recognizable Bragg peaks. The neutron scattering experiments were done at the time-of-flight spectrometer NEAT at Helmholtz Zentrum Berlin [18] using two experimental configurations. The first one with incoming neutron wavelength $\lambda_{I}=2 \AA$ was used to probe the polycrystalline diffraction patterns, which confirmed the formation of the clathrates. The second configuration with $\lambda_{I}=5.1 \AA$ 
and instrumental elastic resolution ranging between 90 and $110 \mu \mathrm{eV}$ was used for investigation of the dynamics in the low energy range of a few $\mathrm{meV}$, corresponding to the picosecond time scale. The spectra, collected in the temperature range from $10-50 \mathrm{~K}$, were corrected using standard data treatment routines. In addition, for the data analysis we excluded detector areas where we observed Bragg reflections from the clathrate framework.

The (incoherent) neutron scattering cross section of $\mathrm{H}_{2}$ molecules is very different for the ortho and para spin isomer states. In the experiment we verified the concentration of ortho-hydrogen by periodically cooling down the sample to $10 \mathrm{~K}$ and measuring the scattering intensities. We found the signal unchanged over the duration of the experiment, confirming our earlier observation of slowdown of ortho to para hydrogen conversion due to confinement [19].

The signal of hydrogen confined in small cages was determined as the difference between the spectra of tetrahydrofuran clathrate and hydrogen filled tetrahydrofuran clathrate. Afterwards this contribution was subtracted from the signal of hydrogen in pure hydrogen clathrates to establish the scattering function of the hydrogen in large cages. The analysis of spectral intensities of hydrogen in large cages shows, that on average two hydrogen molecules occupy a large cage.

\section{Results and analysis}

The melting and boiling temperatures of bulk hydrogen at ambient pressure are $13.99 \mathrm{~K}$ and 20.27 $\mathrm{K}$, respectively [20]. In the frozen state the spectra of the bulk hydrogen at $\mathrm{T}=10 \mathrm{~K}$ show an elastic line only, centered around $\omega=0 \mathrm{meV}$ (Fig. 2a). With melting of hydrogen the elastic line is transformed into a broad Lorentzian due to diffusive motion. Above the boiling point bulk hydrogen is a gas, which leads to faster dynamics and a strong decrease of the signal within our energy / time window.
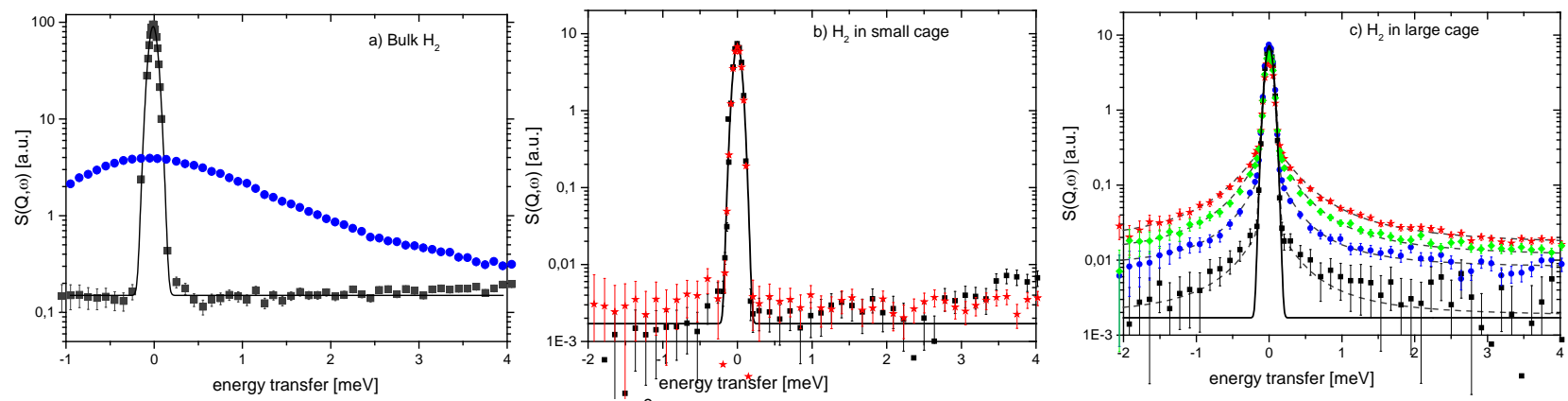

Fig. 2 Dynamic structure factor at $Q=1 \AA^{-1}$ of molecular hydrogen in bulk (a), confined in small (b) and large (c) cages. The solid lines represent the instrumental resolution, which was measured independently with a standard elastic scatterer. Points show experimental data at the selected temperatures: $\mathbf{\square}: \mathrm{T}=10 \mathrm{~K}, \boldsymbol{O}: \mathrm{T}=20 \mathrm{~K}, \diamond: \mathrm{T}=30 \mathrm{~K}, \star$ : $\mathrm{T}=50 \mathrm{~K}$. Dashed lines show fits by the model described in the text. Pronounced quasi-elastic signal of hydrogen confined in the large cages can be observed even at $\mathrm{T}=10 \mathrm{~K}$ indicating remarkable molecular mobility. While the quasi-elastic intensity increases strongly with increasing temperature, its energy width does not change for $\mathrm{T}=10-30 \mathrm{~K}$, and slightly increases at $\mathrm{T}=50 \mathrm{~K}$. The width of quasi-elastic signal is also rather constant in the experimentally covered $\mathrm{Q}$ range, revealing spatially confined diffusion.

Confinement imposes dramatic changes on hydrogen behavior and varies strongly depending on the confinement dimensions [21]. $\mathrm{H}_{2}$ confined into small cages exhibits no additional signal to the intense elastic line up to $50 \mathrm{~K}$, the highest temperature studied here (Fig. 2b). This indicates strongly restricted dynamic activity of $\mathrm{H}_{2}$ in small cages, where $\mathrm{H}_{2}$ is practically "frozen" on the time of this study, localized at an equilibrium position in the center of the cage, cf. Fig. 1. In contrast to small cages and the bulk hydrogen, spectra of $\mathrm{H}_{2}$ confined in large cages reveal high dynamic activity and show a strong quasi-elastic (QENS) signal already at T=10 K (Fig. 2c).

In order to evaluate the QENS signal of confined $\mathrm{H}_{2}$, contributions of various types of motion have to be taken into account, the result of which can be expressed as a convolution: 


$$
S(Q, \omega)=S_{\text {vibrations }}(Q, \omega) \otimes S_{\text {rotations }}(Q, \omega) \otimes S_{\text {diffusion }}(Q, \omega)
$$

In the temperature and energy range studied here, vibrations contribute to the spectra essentially through the Debye-Waller factor only. The inelastic rotation contribution consists of the spectrum of transitions between quantized rotational states of the $\mathrm{H}_{2}$ molecules $\mathrm{J}=0,1, \ldots$, the first of which above the $\mathrm{J}=0$ ground state occurring at $\mathrm{E}=14.7 \mathrm{meV}$, i.e. outside the energy range studied in this experiment. The $\mathrm{J}=0$ elastic component for the ground state introduces a form factor, that is close to 1 for $Q<2 \AA^{-1}[22]$.

The description of $S_{\text {diffusion }}(Q, \omega)$ is more intricate. The quasielastic signal caused by continuous translational diffusion is a Lorentzian with a $Q^{2}$ dependent width at small $Q$ values. The presence of interactions between the diffusing molecule and its environment leads to more complex diffusion mechanisms [23], which lead generally to the sum of an elastic term $A_{0}(Q) \delta(\omega)$ if the diffusive motion is confined to a finite volume on the time scale $t \sim 1 / \omega_{R}$ and one or more Lorentzian quasielastic lines $L(\Gamma, \omega)=\frac{1}{\pi} \frac{\Gamma(Q)}{\omega^{2}+(\Gamma(Q))^{2}}$. Here $\omega_{R}$ is the instrumental energy resolution and $A_{0}(Q)$ the so-called elastic incoherent structure factor (EISF) that reflects the geometrical parameters of the diffusive motion, and $\Gamma(\mathrm{Q})$ is related to the residence times $\tau_{s}$ at the positions of "rest" between jumps between an ensemble of such sites.

We experimentally determined $S_{\text {diffusion }}(Q, \omega)$ by using Eq. 1 for correction to the rotational form factor for $J=0$. For further analysis we tested several models: diffusion on the surface and in the volume of a sphere, jumps between to rest sites (dumb-bell), etc. The best fit was observed using the model of intra-cage jump diffusion between different sorption sites placed at the corners of a tetrahedron, the arrangement suggested by the neutron diffraction study [4] and illustrated in Fig. 1. According to the model developed first for xylenes in zeolites [24], the hydrogen rests on such an adsorption site for the residence time $\tau_{\mathrm{s}}$ and jumps toward another site during a time interval much shorter than the residence time. Considering the occupation of large cages as the average number of two molecules, the dynamic structure factor for this case of diffusive $\mathrm{H}_{2}$ mobility is given by:

$$
S(Q, \omega)=A_{0}(Q) \delta(\omega)+A_{1}(Q)\left[L\left(\frac{2}{\tau_{s}}, \omega\right)+\frac{1}{2} L\left(\frac{4}{\tau_{s}}, \omega\right)\right]+B(Q)
$$

where $A_{0}(Q)=\frac{1}{4}\left[1+3 j_{0}(Q l)\right]$ is the elastic incoherent structure factor EISF for this type of confinement, $A_{1}(Q)=\frac{1}{2}\left[1-j_{0}(Q l)\right]$ is the amplitude of the quasi-elastic scattering contribution, $j_{0}$ is a spherical Bessel function, $1 / \tau_{s}$ is the probability of jump toward a neighboring vacancy and $l$ indicates the jump distance. $B(Q)$ is the constant ( $\omega$ independent) background.

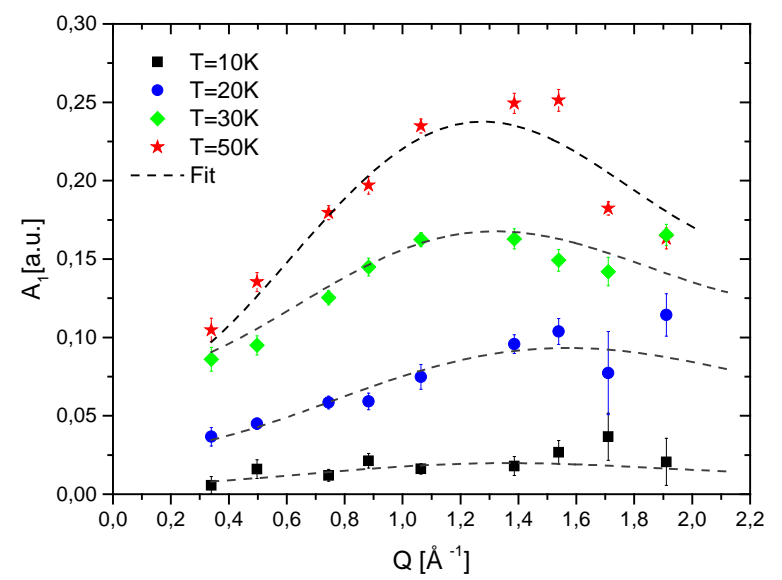

Fig. 3 Amplitude of quasi-elastic scattering signal of molecular hydrogen confined in a large cage of clathrate hydrate. Experimental data are represented by points, dashed lines show fits at different temperatures by the model of jump diffusion within the tetrahedral cluster (Eq. 2), with the fraction of participating mobile $\mathrm{H}_{2}$ molecules increasing with temperature, as described in the text.

We have found that the observed elastic signal was considerable larger than the term $A_{0}(Q)$ obtained by fitting Eq. 2, and the additional $Q$ independent contribution $K_{\text {immobile }} \delta(\omega)$ indicated that a fraction of the $\mathrm{H}_{2}$ molecules did not show mobility on our experimental time scale. With reference to the total scattering intensity at each temperature this allowed us to determine the mobile 
fraction as $K_{\text {mobile }}=1-K_{\text {immobile }}$, whose diffusive mobility is reflected by the quasi-elastic signal intensity in Fig. 3.

Using the set of four parameters - i.e. the fraction of mobile particles, the jump length $l$, the residence time $\tau_{s}$ and the flat background baseline $B(Q)$ - we were able to reproduce all spectra in the $(Q, \omega)$ range covered experimentally (cf. Fig. 2c and Fig. 3). While confinement of hydrogen in small cages leads to the localization of the hydrogen in the center of the cage and a strong slowdown of the dynamics, the confinement in the large cage leads to the formation of novel, more mobile state of hydrogen with enhanced heterogeneous dynamic fluctuations even at our lowest temperature of $10 \mathrm{~K}$. While one fraction of the confined hydrogen is immobile on the time scale of the experiment, the mobile portion of the particles undergo jumps between the tetrahedrally positioned sorption sites with a jump length that agrees within error with the $2.93 \AA$ separation length of the $\mathrm{H}_{2}$ positions suggested by the diffraction studies [4] and is significantly shorter than the $\mathrm{H}_{2}-\mathrm{H}_{2}$ distance of 3.776 $\AA$ in solid hcp hydrogen crystal at ambient pressure [25]. The jump length as well the residence time $\tau_{s}$ shows surprisingly weak temperature dependence (cf. Table 1 ). The increase of the QENS signal with temperature (cf. Fig. 2c) is mostly due to the increased number of mobile particles, which at 50 $\mathrm{K}$ is an order of magnitude larger than at $10 \mathrm{~K}$. Similar dynamic heterogeneity imposed by the local environment was observed previously for hydrogen confined in carbon nanohorns [26].

Table 1. Fraction of mobile molecules, jump length and residence time determined for different temperatures for the intra large cage hydrogen diffusion model Eq. 2

\begin{tabular}{ccccc}
\hline \hline $\begin{array}{c}\text { Temperature } \\
{[\mathrm{K}]}\end{array}$ & $\begin{array}{c}\text { Mobile frac- } \\
\text { tion [\%] }\end{array}$ & $\begin{array}{c}\text { Jump length } l \\
{[\AA]}\end{array}$ & $\begin{array}{c}\text { Residence } \\
\text { time } \tau_{s}[\mathrm{ps}]\end{array}$ & $\begin{array}{c}\text { Baseline } B(Q) \\
\text { in Fig. 2c }\end{array}$ \\
\hline 10 & $4 \pm 1$ & $2.4 \pm 0.6$ & $6.0 \pm 0.13$ & 0.0018 \\
20 & $16 \pm 1$ & $2.4 \pm 0.2$ & $6.1 \pm 0.19$ & 0.007 \\
30 & $21 \pm 2.2$ & $3.22 \pm 0.2$ & $6.3 \pm 0.16$ & 0.011 \\
50 & $36.5 \pm 2.2$ & $3.6 \pm 0.3$ & $4.4 \pm 0.13$ & 0.017 \\
\hline \hline
\end{tabular}

\section{Conclusions}

Our observations can be explained by the modulation of cage potentials as a function of the cage dimension and resulting variation in host- guest interactions. Localization of hydrogen in small cages indicates the existence of potential minima in the center of the cage, matching the molecular size. Indeed, detailed $5 \mathrm{D}$ quantum calculations [27] revealed similar deep minima with a width of $1.5 \AA$ in the potential energy surfaces of small cages. One may argue that such potential can serve as a molecular trap for the confined hydrogen, supporting the reduction of sorption pressure and increasing the release temperature of the confined hydrogen. The increase of the cage dimension leads to a flatter potential, with the existence of four sorption sites, indicating several potential minima displaced from the cage center. Calculated PES profiles in large cage show several minima placed at $\mathrm{d}=3.5 \AA$ from each other and separated by a relatively low potential barrier.

In sum, these results reveal clearly that by varying the size of the cages we can modify the diffusive mobility of confined hydrogen in both directions, i.e. both reducing and enhancing mobility compared to the bulk at the same temperature. In smaller cages of clathrate hydrates the confinement at all temperatures reduces diffusive mobility by orders of magnitude compared to the bulk. This can contribute increased thermal stability for storage by keeping the $\mathrm{H}_{2}$ away from the cage surface. We have also found in contrast that confinement in large cages can enhance the diffusive jump mobility of the hydrogen molecules compared to the bulk solid at the same temperature. The remarkable glassy feature of dynamic heterogeneity [28] between mobile and immobile particles can be related to structural disorder of random deformations in the clathrate lattice, as observed in earlier NMR studies [12]. At the same time, these NMR studies in the same temperature range failed to reveal the surprisingly high and dynamically heterogeneous diffusive intra-cage mobility of $\mathrm{H}_{2}$ molecules below the freezing temperature of bulk hydrogen. 


\section{References}

[1] Y.A. Dyadin, E.G. Larionov, A.Y.Manakov, Mendeleev Commun. 5, (1999) 209.

[2] E.D. Sloan, C.A. Koh, Clathrate Hydrates of Natural Gases, CRC Press, Boca Raton (2008).

[3] W.L. Mao, H.K. Mao, A.F. Goncharov et al, Science 297 (2002) 2247.

[4] K.A. Lokshin,Y. Zhao. D. He et al, Phys. Rev. Lett., 93 (2004) 125503.

[5] H. Lee, J.W.Lee, D.Y. Kim, J. Park et al, Nature, 434 (2005) 743.

[6] G.L. Squires, Introduction to the Theory of Thermal Neutron Scattering, third ed., Cambridge University Press, Cambridge, 2012.

[7] L. Ulivi, M.Celli, A. Giannasi, Phys. Rev. B, 76 (2007) 161401.

[8] M. Xu, Y. S. Elmatad, F. Sebastianelli, J.W. Moskowitz, and Z. Bačić, 2006, J. Phys. Chem. B. Letters, 110 (2006) 24806.

[9] M. Russina, E. Kemner, M. Cellie, L. Ulivi, F. Mezei, J. Physics, 177, (2009) 012013.

[10] D. Colognesi, M. Celli, L. Ulivi, M. Xu, Z. Bačić, J. Phys. Chem. A,117 (2013) 7314.

[11] S. Alavi, J.A. Ripmeester, Angew. Chem., Int. Ed. 46 (2007) 6102.

[12] L. Senadheera, M.S. Conradi, J. Phys. Chem. B 111 (2007)12097.

[13] T. Okuchi, I.L. Moudrakovski, J.A. Ripmeester, Appl. Phys. Lett. 91 (2007) 171903.

[14] F.M. Mulder, M. Wagemaker, L. van Eijck, G.J. Kearley, Chem. Phys. Chem. 9 (2008) 1331.

[15] T.A. Strobel, E.D. Sloan, C.A. Koh, J. Chem. Phys. 130 (2009) 014506.

[16] E. Pefoute, E. Kemner, J. C. Soetens, et al., J. of Phys. Chem. C, 116 (2012) 16823.

[17] K. Lokshin and Y. Zhao, Applied Phys. Lett. 88 (2006) 131909.

[18] Information on https://www.helmholtz-

berlin.de/media/media/grossgeraete/nutzerdienst/neutronen/instrumente/inst/bensc_v3.pdf

[19] M. Russina, E. Kemner, private communication

[20] Information on http://encyclopedia.airliquide.com/Encyclopedia.asp?GasID=36

[21] M. Russina, E. Kemner and F. Mezei; Sci. Rep. 6 (2016) 27417.

[22] P.A. Georgiev, D.K. Ross, A. De Monte et al, Carbon 43 (2005) 895.

[23] R. Hempelmann, Quasielastic Neutron Scattering and Solid State Diffusion, Oxford University Press, Oxford 2000.

[24] H. Jobic, M. Bee, A. Methivier, J. Combet, Microporous and Mesoporous Mat. 42, (2001) 135.

[25] S.S. Batsanov, A.S. Batsanov, Introduction to Structural Chemistry, Springer Science \& Business Media, Heidelberg, 2012.

[26] F. Fernadnez-Alonso, F.J.Bermejo,C. Cabrilli et al., Phys. Rev. Lett., 98, (2007) 215503.

[27] M. Xu, F. Sebastianelli, Z. Bacic, J. Phys. Chem. A, 113 (2009) 7601.

[28] M. Russina, F. Mezei, R. Lechner, S. Longeville, B. Urban, Phys. Rev. Lett. 84 (2000) 3630. 